\begin{tabular}{|c|c|c|}
\hline & Int.J.Curr.Microbiol.App.Sci (2016) 5(7): 100-116 & \\
\hline & International Journal of Current Microbiology and Applied Sciences & $=$ \\
\hline & ISSN: 2319-7706 Volume 5 Number 7 (2016) pp. 100-116 & \\
\hline $\begin{array}{l}\text { EXCELLENT } \\
\text { PUBLISHERS }\end{array}$ & & Inww:ijemas.com \\
\hline
\end{tabular}

Review Article

http://dx.doi.org/10.20546/ijcmas.2016.507.008

\title{
Isolation, Characterization and Antimicrobial Potential of Endophytic Actinomycetes
}

\author{
T.R. Prashith Kekuda ${ }^{1,2 *}$ \\ ${ }^{1}$ Department of Microbiology, Sahyadri Science College (Autonomous), \\ Shivamogga-577203, Karnataka, India \\ ${ }^{2}$ Department of Microbiology, S.R.N.M.N College of Applied Sciences, N.E.S Campus, \\ Balraj Urs Road, Shivamogga-577201, Karnataka, India \\ *Corresponding author
}

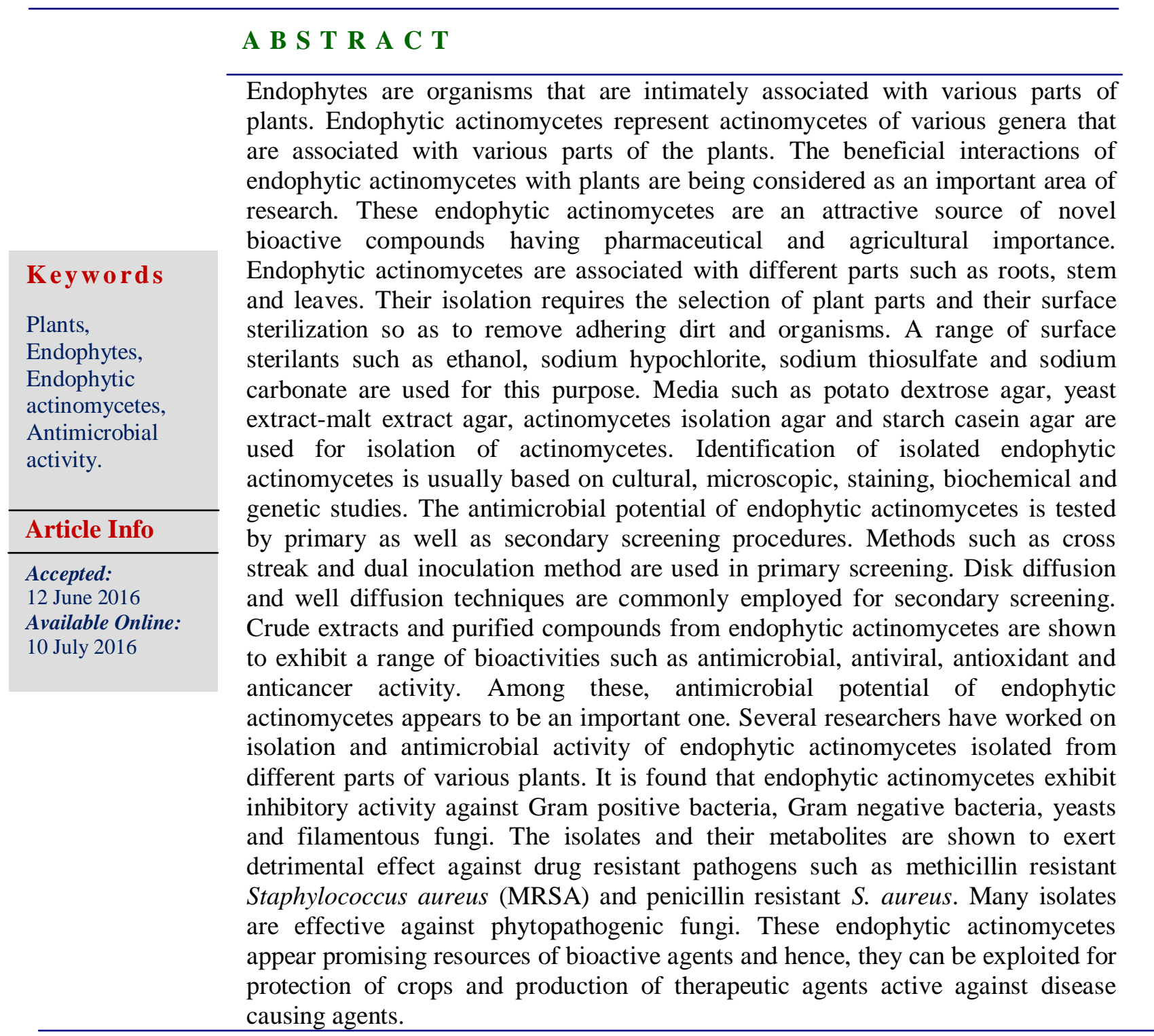




\section{Introduction}

Throughout history, a continuous battle exists between human beings and pathogenic microbes. Infectious diseases caused by bacteria, fungi, viruses and protozoa have been the major cause of morbidity and mortality. Infectious diseases remain the leading cause of death worldwide. Approximately $25 \%$ of annual deaths worldwide are believed to be due to these infectious diseases. Antibiotics form an important group of compounds possessing the property of inhibiting and killing other microorganisms. Antibiotics have been produced by bacteria, fungi and actinomycetes. The antibiotic era began with the accidental discovery of Penicillin (from Penicillium notatum) by Alexander Fleming. Later, Selman Waksman discovered Streptomycin from Streptomyces griseus. The discovery of antibiotics is considered as a turning point in medicine and their use saved countless lives. However, the use of antibiotics has been accompanied by the rapid development of antibiotic resistance in microbes. Methicillin-resistant Staphylococcus aureus (MRSA), multidrugresistant Streptococcus pneumoniae, vancomycin-resistant Enterococcus spp., multidrug-resistant Acinetobacter baumannii, Klebsiella pneumoniae, Escherichia coli, Pseudomonas aeruginosa, multidrug-resistant TB are among the drugresistant pathogens of today. Moreover, the ability of pathogens to acquire and transmit resistance has made the situation even worst and the therapy more complicated. These drug resistant pathogens impose a substantial burden to human population. Therefore, search for new antimicrobials is a continuous process to keep pace with continually evolving pathogens (Castillo et al., 2002; Tenover, 2006; Dzidic et al., 2008; Davies and Davies, 2010, Joseph et al., 2012; Kekuda et al., 2013; Kekuda et al., 2015).
The microbial metabolites form important sources of life saving environments such as bacterial and fungal infections, Cancer, transplant rejection and high cholesterol. There are over 23,000 known microbial secondary metabolites. A major portion of these metabolites are produced by actinobacteria and fungi while bacteria account for remaining portion. Among microbes producing bioactive secondary metabolites, actinomycetes produced highest number of bioactive metabolites (Saadoun and Al-Momani, 2000; Berdy, 2005; Zhao et al., 2006; Zin et al., 2007; Kekuda, 2014). Many microbes such as bacteria, actinomycetes and fungi have been found to be associated with the internal tissues of plant and are known as endophytes (within plants). The term endophyte was coined by De Bary (1866), which involves the existence of microorganisms inside the plant tissues without having negative effects on host plant. Endophytes occupy cortical tissues of roots and confer defense against invading pathogens while the cortex confers protection to endophytes from the harsh environment of the rhizosphere. In general endophytic bacteria occur at lower population densities than rhizospheric bacteria. Almost all the plants have been found to be associated with one or more endophytes. These endophytes have been isolated from both monocots and dicots, ranging from woody tree species to herbaceous crop plants. Endophytes that colonize inner tissues of plants usually obtain nutrition as well as protection from host plants and in return, they provide enhanced fitness to the host by producing bioactive metabolites which protect the plant. These microbes are shown to produce metabolites such as growth promoters, insect and pest repellents, antimicrobials and protectors in stress conditions. Endophytes have the potential to produce unique secondary metabolites, which can be 
exploited in pharmaceutical, agricultural and other industries. Thus, there is a growing interest of researchers in bioprospecting of endophytic microbial communities inhabiting the plants from various ecosystems (Petrini et al., 1992; Rosenblueth and Martínez-Romero, 2006; Zhang et al., 2006; Ryan et al., 2008; Kafur and Khan, 2011; Selim et al., 2012; Taechowisan et al., 2013; Golinska et al., 2015; Passari et al., 2015; Roy and Banerjee, 2015). In this review, methods available for isolation and characterization and evaluation of antimicrobial activity of endophytic actinomycetes strains have been discussed and also literature survey on antimicrobial activity of endophytic actinomycetes recovered from various plants is presented.

\section{Actinomycetes}

Actinomycetes (order Actinomycetales) represents high $\mathrm{G}+\mathrm{C}$ containing, filamentous, Gram positive bacteria that are widespread in nature and distributed in a variety of ecological habitats such as soil, fresh water, marine environment, plants (as endophytes) and others. They are involved in the decomposition of organic matter in soil, including lignin and other recalcitrant polymers, and can degrade agricultural and urban wastes. The actinomycetes and their bioactive metabolites have shown to possess antimicrobial, cytotoxic, plant growth promoting, antiviral, antioxidant, insecticidal, antiprotozoal, anthelmintic, enzyme inhibitory, plant growth promoting and herbicidal agents. They are known to play key role in bioremediation of dyes, petroleum products and pesticides and biosorption of heavy metals. The bioactive metabolites from actinomycetes are also active against antibiotic resistant bacteria and plant pathogenic fungi. Among various genera, the genus Streptomyces seems to be abundant and the members of this genus have produced a wide range of metabolites having several bioactivities (Cao et al., 2004; Zin et al., 2007; Bunyoo et al., 2009; Gonzalez-Franco et al., 2009; Kekuda et al., 2010; Qin et al., 2011; Kumar and Krishnan, 2012; Kekuda et al., 2013; Taechowisan et al., 2013).

\section{Endophytic Actinomycetes and their Potential Roles}

Actinomycetes of various genera are found associated with plants. The beneficial interactions of endophytic actinomycetes with plants are being considered as an important area of research. These endophytic actinobacteria are attractive source of novel bioactive compounds and therefore, many research groups are involved in the study of their bioactivities and industrial applications. It is experimentally proven that the endophytic actinomycetes display a wide array of bioactivities. Crude solvent extracts and purified compounds from endophytic actinomycetes are shown to exhibit bioactivities such as antimicrobial, antiviral, antioxidant, antitumor, larvicidal, antimalarial, antidiabetic and plant growth promotory activities (Table 1). The studies have also shown that the endophytic actinomycetes exhibit inhibitory activity against drug resistant pathogens (Castillo et al., 2002; Qin et al., 2011; Joseph et al., 2012; Huang et al., 2012; Golinska et al., 2015; Savi et al., 2015; Passari et al., 2015; Singh and Dubey, 2015).

\section{Location of Endophytic Actinomycetes}

Endophytic actinomycetes are shown to associate with their host at a very early stage of the plant development (Minamiyama et al., 2003; Hasegawa et al., 2006). Endophytic actinomycetes have been 
isolated from various parts of the plants. The woody plants conferred far greater diversity of actinomycetes in comparison to herbaceous plants. In most cases, the maximum endophytic actinobacteria have been recovered from roots followed by stems and leaves. The high rate of occurrence of actinomycetes in roots is due to the fact that the actinomycetes are natural dwellers of soil and hence easily come in contact with the roots of plants and may form the symbiotic association with them by entering the plant tissues. The greater diversity of endophytes is probable to occur in the tropical and temperate regions. The genus Streptomyces seems to be relatively abundant genus (Strobel and Daisy, 2003; Qin et al., 2009; Nimnoi et al., 2010; Huang et al., 2012; Gangwar et al., 2014; Golinska et al., 2015; Rao et al., 2015; Passari et al., 2015).

\section{Isolation of Endophytic Actinomycetes}

Searching specific endophytes capable of producing antimicrobials is not necessarily a random process. The first objective is to select one or more plants that may harbor endophyte. This selection process is usually done on the basis of the environment and the age of a plant. Besides, the ethomedicinal uses of the plants also form a basis for selection of plants (Castillo et al., 2002). Several methods are employed for isolation of endophytic actinobacteria from different plants. The recovery of diverse actinobacteria depends mainly on the methods of isolation. The most commonly employed method for their detection and enumeration involves isolation from surfacesterilized plant tissue. Various factors such as host plant species, age and type of tissue, geographical and habitat distribution, sampling season, surface sterilants, selective media and culture conditions influence isolation of endophytic actinobacteria
(Castillo et al., 2002; Takahashi and Omura, 2003; Kafur and Khan, 2011; Golinska et al., 2015).

\section{Surface Sterilization of Plant Parts}

The key as well as obligatory step that is to be followed after selection of plants and their parts such as leaves, stem and roots for isolation of endophytic actinomycetes is to eliminate the microorganisms that are present on the surface. This is accomplished by surface sterilization of plant parts. These explants are washed in running tap water to remove adhered microbes, soil debris or dust particles, followed by surface sterilization using one or more different surface sterilants. The most frequently used surface sterilants are ethanol and a strong oxidant or general disinfectant like household bleach $(\mathrm{NaOCl})$ with $2-5 \%(\mathrm{w} / \mathrm{v})$ available chlorine (for 2-4 min). Besides, combination of $5 \%$ sodium chlorate $\left(\mathrm{NaClO}_{3}\right), 2.5 \%$ sodium thiosulfate $\left(\mathrm{Na}_{2} \mathrm{~S}_{2} \mathrm{O}_{3}\right), 75 \%$ ethanol and $10 \%$ sodium bicarbonate $\left(\mathrm{NaHCO}_{3}\right)$ were also used as sterilizing agents. Ethanol concentrations of $70 \%$ and $95 \%$ have also been used. Hydrogen peroxide has been used for surface sterilization. Surfactants (wetting agents) such as Tween 20, Tween 80 and Triton X-100 can also be added so as to enhance the effectiveness of surface sterilization. Addition of sodium thiosulfate is often recommended as thiosulfate can suppress the detrimental effect of residual $\mathrm{NaOCl}$ on surface of explant, which may kill endophytes or does not allow endophytes to form colonies. Soaking of plant tissues in $10 \% \mathrm{NaHCO}_{3}$ solution inhibit the endophytic fungi, which could overgrow and mask the actinobacteria. The strength of sterilizing chemicals depends on permeability of the sample. Otherwise, the internal tissues will be sterilized. Finally, the explants are rinsed with sterile distilled water, divided into small pieces and 
inoculated on appropriate agar medium. Alternatively, the surface sterilized plant tissues can be macerated and thoroughly homogenized with phosphate buffer or other suitable liquid medium. This suspension is serially diluted and plated on respective media. The efficacy of the surface sterilization, resulting from lack of microbial growth, can be authenticated by inoculating the last washing water into the same media plates (Cao et al., 2004; Zin et al., 2007; Ghadin et al., 2008; Qin et al., 2009; Qin et al., 2011; Passari et al., 2015; Golinska et al., 2015).

\section{Media used for Isolation of Endophytic Actinomycetes}

Several growth media are used by various researchers for the isolation of actinomycetes from plant samples. Media such as Starch casein agar, Starch casein nitrate agar, Humic acid-Vitamin agar, Yeast extract-Malt extract agar, Actinomycetes isolation agar, Potato dextrose agar, Glycerol asparagine salts agar and Tap water-Yeast extract agar are frequently used for isolation of endophytic actinomycetes. The growth media are usually amended with antibiotics such as Nystatin, Nalidixic acid and Cycloheximide which inhibit the growth of fungi and other bacteria (Ghadin et al., 2008; Qin et al., 2011; Golinska et al., 2015; Passari et al., 2015).

It is shown that medium containing low nutrients (for e.g. tap water yeast extract agar) seems to be effective for isolation of endophytic actinomycetes, due to the fact that medium with high nutrient concentration allow the growth of fast growing bacteria which may overgrow slow growing actinomycetes (Qin et al., 2011). Table 2 depicts growth media used by various researchers for isolation of endophytic actinomycetes from different plants.

\section{Identification of Endophytic Actinomycetes}

At present, the identification of actinomycetes is based on polyphasic approach comprising morphological, physiological and molecular studies. Based on these features, each taxon should be described and differentiated from related taxa. Distinguishing phenotypic differences are required for the description of a new species. Characteristics of colonies, the presence of aerial mycelia and substrate mycelia, spore mass color, distinctive reverse colony color and production of diffusible pigment, and sporophore and spore chain morphology are carried out. Electron microscopy is very useful in studyin the morphology of hyphae and spores. Staining characteristics and the presence of various enzymes are detected by biochemical tests. Requirement of $\mathrm{pH}$, temperature and utilization of various carbohydrates and amino acids are also screened. Determination of cell wall types and characteristic sugars are also noted. The sequencing of highly conserved macromolecules, notably $16 \mathrm{~S}$ rRNA genes, has provided valuable data for constructing phylogenies at and above the genus level.

The DNA:DNA relatedness, molecular fingerprinting and phenotypic techniques are methods of choice for delineating taxa at and below the rank of species (Shirling and Gottlieb, 1966; Wayne et al. 1987; Rossello'-Mora and Amann 2001; Ezra et al., 2004; Zin et al., 2007; Qin et al., 2009; Goodfellow et al. 2012; Golinska et al., 2015; Passari et al., 2015). Table 3 shows various parameters used by researchers for identification of isolated actinomycetes. 


\section{Methods used to Evaluate Antimicrobial activity of Endophytic Actinomycetes}

Several protocols have been used to evaluate antimicrobial potential of endophytic actinomycetes. Methods such as cross streak technique and dual inoculation are used for preliminarily screening for antimicrobial potential of actinomycetes (Gayathri and Muralikrishnan, 2013; Kandpal et al., 2013; Phuakjaiphaeo and Kunasakdakul, 2015; Passari et al., 2015; Rao et al., 2015).

Methods such as disk diffusion and agar well diffusion are performed to investigate antimicrobial potential of crude solvent extracts and purified compounds (Castillo et al., 2002; El-Shatoury et al., 2006; Li et al., 2008; Khafur and Khan, 2011; Singh and Padmavathy, 2015; Saini et al., 2016). Some authors have employed micro-broth dilution technique (Castillo et al., 2002; Mai et al., 2013). Bioautographic method has been used to evaluate inhibitory efficacy of separated components (Savi et al., 2015).

\section{Antimicrobial activity of endophytic actinomycetes}

Several species of endophytic actinomycetes exhibit a range of bioactivities which can be exploited commercially. Among bioactivities shown by these endophytes, antimicrobial efficacy seems to be the best one. Crude extracts and purified compounds from endophytic strains of actinomycetes have shown to inhibit a wide range of pathogenic microorganisms of plants and animals including drug resistant pathogens. In a study, $43.4 \%$ of isolates of endophytic actinomycetes recovered from leaves and roots of maize showed antimicrobial activity against one or more tested bacteria and yeast (de Araújo et al., 2000). 55\% of endophytic actinomycetes recovered from leaves of Paeonia lactiflora and Trifalium repens inhibited the growth of Rhizoctonia solani, a significant pathogen of plants ( $\mathrm{Gu}$ et al., 2006).

The ethyl acetate extract of Streptomyces strain SUK 06 recovered from Thottea grandiflora showed promising antimicrobial activity against several bacterial strains including MRSA and phytopathogenic fungi (Ghadin et al. 2008). Endophytic actinomycetes isolated from pharmaceutical plants in Yunnan province, China exhibited inhibitory activity against Gram positive and Gram negative bacteria (Li et al., 2008). A strain designated as Streptomyces sp. Tc052 isolated from roots of Alpinia galanga was shown to possess strong antimicrobial activity with MIC value of $64-128 \mu \mathrm{g} / \mathrm{ml}$ (Taechowisan et al., 2008). An endophytic strain designated as CEN26, isolated from Centella asiatica, displayed high inhibitory activity against Alternaria brassicicola and resulted in morphological disorders of the pathogen, swollen hyphae and frequent septa of the treated pathogen mycelia (Phuakjaiphaeo and Kunasakdakul, 2015). Further, Table 4 reveals the antimicrobial activity of endophytic actinomycetes recovered from different plants by various researchers.

\section{Endophytic actinomycetes against drug resistant pathogens}

It has been shown that endophytic actinomycetes and their metabolites have the capability to inhibit drug resistant pathogenic microorganisms. Munumbicins A, B, C and D were isolated from Streptomyces NRRL 3052 (an endophyte in the stem of medicinal plant Kennedia nigriscans) demonstrated inhibitory activity against multidrug-resistant Mycobacterium tuberculosis (Castillo et al., 2002). The ethyl acetate extract of Streptomyces strain SUK 06 recovered from Thottea grandiflora showed promising antibacterial activity against MRSA (Ghadin et al. 2008). In a 
study, 12 out of 65 strains of endophytic actinomycetes recovered from Achyranthes bidentata, Paeonia lactiflora, Radix platycodi and Artemisiae argyi have shown inhibitory activity against penicillin-resistant staphylococcus aureus (Zhang et al., 2012).

Table.1 Some bioactivities of endophytic actinomycetes

\begin{tabular}{|c|c|c|}
\hline Endophyte & Bioactivity & Reference \\
\hline Streptomyces NRRL 30562 & Antimicrobial, antimalarial & Castillo et al. (2002) \\
\hline Streptomyces sp. Tc052 & Antioxidant & Taechowisan et al. (2009) \\
\hline Streptomyces aureofaciens CMUAc130 & Antifungal & Taechowisan et al. (2005) \\
\hline S. aureofaciens CMUAc130 & Anti-inflammatory & Taechowisan et al. (2007) \\
\hline S. aureofaciens CMUAc130 & Antitumor & Taechowisan et al. (2007) \\
\hline Endophytic streptomycetes & Antitumor, antimicrobial & Li et al. (2008) \\
\hline Streptomyces sp. 34 and Leifsonia xyli 24 & Plant growth promotion & Passari et al. (2015) \\
\hline Streptomyces sp. & $\begin{array}{l}\text { Plant growth promotion and } \\
\text { disease control }\end{array}$ & Sreeja and Gopal (2013) \\
\hline $\begin{array}{l}\text { Streptomyces albovinaceus and } \\
\text { S. badius }\end{array}$ & Insecticidal & Tanvir et al. (2014) \\
\hline Strain BWA65 & Alpha-glucosidase inhibitor & Pujiyanto et al. (2012) \\
\hline Streptomyces sp. & Antioxidant & Zhou et al. (2014) \\
\hline Micromonospora lupine sp. nov. & Antitumor & Igarashi et al. (2007) \\
\hline
\end{tabular}

Table.2 Media used by various researchers to isolate endophytic actinomycetes

\begin{tabular}{|c|c|}
\hline Medium used & Reference \\
\hline Inhibitory Mold Agar-2 (IMA-2) & Phuakjaiphaeo and Kunasakdakul (2015) \\
\hline Yeast extract agar and Yeast extract- Malt extract agar & Piza et al. (2015) \\
\hline Potato dextrose agar & Mai et al. (2013), Savi et al. (2015) \\
\hline $\begin{array}{l}\text { Starch casein nitrate agar, Actinomycetes isolation agar, } \\
\text { Tap water yeast extract agar, Yeast malt extract agar } \\
\text { and Glycerol asparagine agar }\end{array}$ & Passari et al. (2015), Roy and Banerjee (2015) \\
\hline $\begin{array}{l}\text { Starch casein agar, Actinomycetes isolation agar, Tap } \\
\text { water Yeast extract agar, Tryptone soya agar, Potato } \\
\text { dextrose agar, CYC agar, Arginine glycerol salts agar }\end{array}$ & El-Shatoury et al. (2006) \\
\hline Tryptic soy agar & Gangwar et al. (2014) \\
\hline S medium & Cao et al. (2004) \\
\hline ATCC 172 agar & Huang et al. (2012) \\
\hline Glucose-asparagine agar & Huang et al. (2012) \\
\hline Water agar & Ezra et al. (2004) \\
\hline Humic acid-Vitamin agar & $\begin{array}{l}\text { Taechowisan et al. (2008), Huang et al. } \\
\text { (2012), Sunaryanto and Mahsunah (2013) }\end{array}$ \\
\hline Organic medium 2 Gause & Machavariani et al. (2014) \\
\hline Soya bean agar & Shenpagam et al. (2012) \\
\hline Starch casein agar & $\begin{array}{l}\text { Zin et al. (2007), Ghadin et al. (2008), } \\
\text { Bunyoo et al. (2011), Kafur and Khan (2011), } \\
\text { Kandpal et al. (2012), Vijayan et al. (2014), } \\
\text { Saini et al. (2016) }\end{array}$ \\
\hline
\end{tabular}


Table.3 Methods used for characterization of endophytic actinomycetes

\begin{tabular}{|c|c|c|c|}
\hline Host plant & Strain(s) & Characterization & Reference \\
\hline Monstera sp. & $\begin{array}{l}\text { Streptomyces sp. (MSU- } \\
2110)\end{array}$ & $\begin{array}{l}\text { Microscopic and } 16 \mathrm{~S} \text { rDNA } \\
\text { gene sequencing }\end{array}$ & $\begin{array}{l}\text { Ezra et al. } \\
(2004)\end{array}$ \\
\hline $\begin{array}{l}\text { Paeonia } \\
\text { lactiflora and } \\
\text { Trifalium repens }\end{array}$ & $\begin{array}{l}\text { Pseudonocardia and } \\
\text { streptomycetes }\end{array}$ & $\begin{array}{l}\text { Morphological and } 16 \mathrm{~S} \\
\text { rRNA gene sequencing }\end{array}$ & Gu et al. (2006) \\
\hline $\begin{array}{l}\text { Thottea } \\
\text { grandiflora, } \\
\text { Polyalthia spp. } \\
\text { And } \\
\text { Mapania sp. }\end{array}$ & Streptomycetes & 16S rRNA gene sequencing & $\begin{array}{l}\text { Zin et al. } \\
(2007)\end{array}$ \\
\hline $\begin{array}{l}\text { Thottea } \\
\text { grandiflora }\end{array}$ & Streptomyces SUK 06 & $\begin{array}{l}\text { The morphological and } 16 \mathrm{~S} \\
\text { rRNA sequence analysis }\end{array}$ & $\begin{array}{l}\text { Ghadin et al. } \\
\text { (2008) }\end{array}$ \\
\hline Alpinia galanga & Streptomyces sp. & $\begin{array}{l}\text { Morphology and amino acid } \\
\text { composition of whole cell } \\
\text { extract }\end{array}$ & $\begin{array}{l}\text { Taechowisan et } \\
\text { al. (2008) }\end{array}$ \\
\hline $\begin{array}{l}\text { Acacia } \\
\text { auriculiformis }\end{array}$ & $\begin{array}{l}\text { Streptomyces, } \\
\text { Actinoallomurus, } \\
\text { Amycolatopsis, Kribbella } \\
\text { and Microbispora }\end{array}$ & 16S rRNA sequencing & $\begin{array}{l}\text { Bunyoo et al. } \\
\text { (2011) }\end{array}$ \\
\hline Artemisia anпиа & $\begin{array}{l}\text { Pseudonocardia } \\
\text { antimicrobica } \mathrm{sp} . \text { nov. }\end{array}$ & $\begin{array}{l}\text { Cell-wall peptidoglycan, fatty } \\
\text { acid, G+C content, 16S rRNA } \\
\text { gene sequence, DNA-DNA } \\
\text { relatedness, }\end{array}$ & $\begin{array}{l}\text { Zhao et al. } \\
\text { (2012) }\end{array}$ \\
\hline $\begin{array}{l}\text { Curcuma } \\
\text { domestica }\end{array}$ & $\begin{array}{l}\text { Streptomyces strain } \\
\text { KY01 }\end{array}$ & $\begin{array}{l}\text { Morphological and } 16 \mathrm{~S} \\
\text { rRNA gene sequencing }\end{array}$ & $\begin{array}{l}\text { Sunaryanto and } \\
\text { Mahsunah } \\
(2013)\end{array}$ \\
\hline $\begin{array}{l}\text { Zingiber } \\
\text { officinale }\end{array}$ & Streptomyces sp. GMT-8 & $\begin{array}{l}\text { Morphology, } \\
\text { chemotaxonomy and 16S } \\
\text { rDNA sequencing }\end{array}$ & $\begin{array}{l}\text { Taechowisan et } \\
\text { al. (2013) }\end{array}$ \\
\hline Ocimum sanctum & Nocardiopsis species & $\begin{array}{l}\text { Morphological and } 16 \mathrm{~S} \\
\text { rRNA gene sequencing }\end{array}$ & $\begin{array}{l}\text { Singh and } \\
\text { Padmavathy } \\
(2015)\end{array}$ \\
\hline $\begin{array}{l}\text { Macleaya } \\
\text { cordata }\end{array}$ & $\begin{array}{l}\text { Streptomyces } \\
\text { mobaraensis }\end{array}$ & $\begin{array}{l}\text { Morphological, biochemical } \\
\text { and molecular studies }\end{array}$ & $\begin{array}{l}\text { Wang et al. } \\
(2014)\end{array}$ \\
\hline $\begin{array}{l}\text { Lycopersicon } \\
\text { esculentum }\end{array}$ & Streptomyces & $\begin{array}{l}\text { Morphological and cultural } \\
\text { characteristics }\end{array}$ & $\begin{array}{l}\text { Sreeja and } \\
\text { Gopal (2013) } \\
\end{array}$ \\
\hline $\begin{array}{l}\text { Vochysia } \\
\text { divergens }\end{array}$ & $\begin{array}{l}\text { Microbispora } \mathrm{sp} ., \\
\text { Streptomyces sampsonii, } \\
\text { Micromonospora } \mathrm{sp} .\end{array}$ & 16S rRNA sequencing & $\begin{array}{l}\text { Savi et al. } \\
(2015)\end{array}$ \\
\hline Cinnamomum sp. & Streptomyces rochei & $\begin{array}{l}\text { Morphological, physiological, } \\
\text { biochemical and 16S rRNA } \\
\text { gene analysis }\end{array}$ & $\begin{array}{l}\text { Roy and } \\
\text { Banerjee (2015) }\end{array}$ \\
\hline
\end{tabular}


Int.J.Curr.Microbiol.App.Sci (2016) 5(7): 100-116

Table.4 Antimicrobial activity of endophytic actinomycetes

\begin{tabular}{|c|c|c|c|}
\hline Host plant & Strain(s) & Activity against & Reference \\
\hline $\begin{array}{l}\text { Thottea grandiflora, } \\
\text { Polyalthia spp. and } \\
\text { Mapania sp. }\end{array}$ & Streptomycetes & Phytopathogenic fungi & Zin et al. (2007) \\
\hline Eucalyptus globus & Streptomyces & $\begin{array}{l}\text { Gram negative } \\
\text { bacteria }\end{array}$ & $\begin{array}{l}\text { Muthiah et al. } \\
(2009)\end{array}$ \\
\hline Azadirachta indica & Streptomyces and others & Bacteria and fungi & Verma et al. (2009) \\
\hline Acacia auriculiformis & Streptomyces and others & Bacteria and fungi & $\begin{array}{l}\text { Bunyaoo et } a l . \\
(2011)\end{array}$ \\
\hline $\begin{array}{l}\text { Aloe vera, Mentha } \\
\text { arvensis and Ocimum } \\
\text { sanctum }\end{array}$ & Saccharopolyspora 0-9 & Phytopathogenic fungi & $\begin{array}{l}\text { Gangwar et al. } \\
(2011)\end{array}$ \\
\hline $\begin{array}{l}\text { Spermacoce } \\
\text { verticillata }\end{array}$ & $\begin{array}{lll}\text { Streptomyces } & & \text { sp., } \\
\text { Microbispora } & \text { sp. } & \text { and } \\
\text { Nocardia } \text { sp. } & & \\
\end{array}$ & Gram positive bacteria & Conti et al. (2012) \\
\hline $\begin{array}{l}\text { Zingiber officinale and } \\
\text { Alpinia galanga }\end{array}$ & $\begin{array}{l}\text { Streptomyces aureofaciens } \\
\text { CMUAc130 }\end{array}$ & Fungi & $\begin{array}{l}\text { Taechowisan and } \\
\text { Lumyong (2003) }\end{array}$ \\
\hline $\begin{array}{l}\text { Lycopersicon } \\
\text { Esculentum }\end{array}$ & Streptomyces & $\begin{array}{l}\text { Ralstonia } \\
\text { solanacearum }\end{array}$ & $\begin{array}{l}\text { Sreeja and Gopal } \\
(2013)\end{array}$ \\
\hline Zingiber officinale & Streptomyces sp. GMT-8 & $\begin{array}{lr}\text { Gram } & \text { positive and } \\
\text { Gram } & \text { negative } \\
\text { bacteria } & \\
\end{array}$ & $\begin{array}{l}\text { Taechowisan et al. } \\
\text { (2013) }\end{array}$ \\
\hline Sugarcane and banana & $\begin{array}{l}\text { Streptomyces } \\
\text { and others }\end{array}$ & Bacteria and fungi & $\begin{array}{l}\text { Gayathri and } \\
\text { Murulikrishnan } \\
(2013)\end{array}$ \\
\hline Macleaya cordata & Strain BL7 & $\begin{array}{l}\text { Staphylococcus } \\
\text { aureus and Bacillus } \\
\text { subtilis }\end{array}$ & Wang et al. (2014) \\
\hline Glycine max & Strain JDA 1 and JDA 12 & Phytopathogenic fungi & $\begin{array}{l}\text { Dalal and Kulkarni } \\
(2014)\end{array}$ \\
\hline $\begin{array}{l}\text { Neem, eucalyptus and } \\
\text { coffee seeds }\end{array}$ & $\begin{array}{l}\text { Strain NEK5, EE9 and } \\
\text { CE1 }\end{array}$ & Phytopathogenic fungi & Vijayan et al. (2014) \\
\hline Combretum latifolium & Streptomyces and others & Bacteria and fungi & Rao et al. (2015) \\
\hline Vochysia divergens & Strain LGMB259 & $\begin{array}{l}\text { Bacteria, Candida } \\
\text { albicans and MRSA }\end{array}$ & Savi et al. (2015) \\
\hline Centella asiatica & Strain CEN26 & $\begin{array}{l}\text { Alternaria } \\
\text { brassicicola }\end{array}$ & $\begin{array}{l}\text { Phuakjaiphaeo and } \\
\text { Kunasakdakul } \\
\text { (2015) }\end{array}$ \\
\hline Ocimum sanctum & Nocardiopsis No.5 & Bacteria & $\begin{array}{l}\text { Singh and } \\
\text { Padmavathy (2015) }\end{array}$ \\
\hline Cinnamomum sp. & Streptomyces rochei & Bacteria & $\begin{array}{l}\text { Roy and Banerjee } \\
(2015)\end{array}$ \\
\hline Syzygium cumini & Isolate $\mathrm{J}-10$ & $\begin{array}{l}\text { Staphylococcus and } \\
\text { others }\end{array}$ & Saini et al. (2016) \\
\hline $\begin{array}{l}\text { Miconia albicans } \\
\text { (Sw.) Triana }\end{array}$ & Amycolatopsis orientalis & $\begin{array}{l}\text { Bacteria and } \\
\text { C.albicans }\end{array}$ & Pizza et al. (2015) \\
\hline
\end{tabular}


Table.5 Inhibitory activity of purified compounds from endophytic actinomycetes

\begin{tabular}{|c|c|c|c|}
\hline Compound & Endophytic strain & Activity against & Reference \\
\hline 24-demethyl-bafilomycin $\mathrm{C}_{1}$ & Streptomyces sp. CS & Antimicrobial & $\begin{array}{l}\mathrm{Lu} \text { and Shen } \\
(2003)\end{array}$ \\
\hline Cedarmycins A and B & $\begin{array}{lll}\text { Streptomyces } & \text { sp. } & \text { TP- } \\
\text { A0456 } & & \\
\end{array}$ & Antifungal & Igarashi (2004) \\
\hline Celastramycins A and B & $\begin{array}{l}\text { Streptomyces sp. MaB- } \\
\text { QuH-8 }\end{array}$ & Antimicrobial & $\begin{array}{l}\text { Pullen et al. } \\
(2002)\end{array}$ \\
\hline Saadamycin & $\begin{array}{l}\text { Streptomyces } \quad \text { sp. } \\
\text { Hedaya-48 }\end{array}$ & Antifungal & $\begin{array}{l}\text { El-Gendy and } \\
\text { El-Bondkly } \\
(2010)\end{array}$ \\
\hline 24-demethyl-bafilomycin $\mathrm{A}_{2}$ & Streptomyces sp. CS & Antimicrobial & $\begin{array}{l}\text { Lu and Shen } \\
(2004)\end{array}$ \\
\hline Alnumycin & Streptomyces sp. & Gram positive bacteria & $\begin{array}{lll}\text { Birber } & \text { et } & \text { al. } \\
(1998) & & \end{array}$ \\
\hline Dinactin and nonactin & Streptomyces sp. Is9131 & Antimicrobial & $\begin{array}{l}\text { Zhao et al. } \\
(2005)\end{array}$ \\
\hline Antimycin $\mathrm{A}_{18}$ & $\begin{array}{l}\text { Streptomyces } \\
\text { albidoflavus }\end{array}$ & Antifungal & $\begin{array}{l}\text { Yan } \\
(2010)\end{array}$ \\
\hline Demethylnovobiocins & $\begin{array}{lll}\text { Streptomyces sp. } & \text { TP- } \\
\text { A0556 } & & \\
\end{array}$ & Antimicrobial & Igarashi (2004) \\
\hline 6-Prenylindole & $\begin{array}{lll}\text { Streptomyces } & \text { sp. } & \text { TP- } \\
\text { A0595 } & & \\
\end{array}$ & Antifungal & Igarashi (2004) \\
\hline Munumbicins A, B, C and D & $\begin{array}{l}\text { Streptomyces NRRL } \\
3052 \text { (from Kennedia } \\
\text { nigriscans) }\end{array}$ & $\begin{array}{l}\text { Gram positive bacteria and } \\
\text { multidrug-resistant } \\
\text { Mycobacterium tuberculosis }\end{array}$ & $\begin{array}{l}\text { Castillo et al. } \\
\text { (2002) }\end{array}$ \\
\hline Kakadumycin A & $\begin{array}{l}\text { Streptomyces sp. NRRL } \\
30566 \text { (from Grevillea } \\
\text { pteridifolia) }\end{array}$ & Gram-positive bacteria & $\begin{array}{l}\text { Castillo et al. } \\
\text { (2003) }\end{array}$ \\
\hline Coronamycins & $\begin{array}{l}\text { Streptomyces sp.(MSU- } \\
2110)\end{array}$ & Yeasts and molds & $\begin{array}{l}\text { Ezra } \text { et al. } \\
(2004)\end{array}$ \\
\hline $\begin{array}{l}\text { 5,7-dimethoxy-4- } \\
\text { pmethoxylphenylcoumarin (i), } \\
5,7 \text {-dimethoxy-4- } \\
\text { phenylcoumarin (ii), } \\
\text { Vanillin (iii) and 3-methoxy-4- } \\
\text { hydroxytoluene (iv) }\end{array}$ & $\begin{array}{l}\text { Streptomyces } \\
\text { aureofaciens } \\
\text { CMUAc130 (from } \\
\text { Zingiber officinale) }\end{array}$ & $\begin{array}{l}\text { Colletotrichum musae and } \\
\text { Fusarium oxysporum }\end{array}$ & $\begin{array}{l}\text { Taechowisan et } \\
\text { al. }(2005)\end{array}$ \\
\hline Actinomycin D & Streptomyces sp. Tc022 & $\begin{array}{l}\text { Colletotrichum musae and } \\
\text { Candida albicans }\end{array}$ & $\begin{array}{l}\text { Taechowisan et } \\
\text { al. }(2006)\end{array}$ \\
\hline $\begin{array}{l}\text { Kaempferol, Isoscutellarin, } \\
\text { Umbelliferone, Cichoriin }\end{array}$ & $\begin{array}{l}\text { Streptomyces } \mathrm{sp} . \mathrm{Tc} 052 \\
\text { (from Alpinia galanga) }\end{array}$ & $\begin{array}{l}\text { Gram positive and Gram } \\
\text { negative bacteria, Candida } \\
\text { albicans and Colletotrichum } \\
\text { musae }\end{array}$ & $\begin{array}{l}\text { Taechowisan et } \\
\text { al. (2008) }\end{array}$ \\
\hline Decursin & $\begin{array}{l}\text { Streptomyces sp. GMT- } \\
8 \text { (from Zingiber } \\
\text { officinale) } \\
\end{array}$ & $\begin{array}{l}\text { Gram positive and Gram } \\
\text { negative bacteria }\end{array}$ & $\begin{array}{l}\text { Taechowisan et } \\
\text { al. (2013) }\end{array}$ \\
\hline $\begin{array}{l}\text { 3-acetonylidene-7- } \\
\text { prenylindolin-2-one (new } \\
\text { prenylated indole derivative) }\end{array}$ & $\begin{array}{l}\text { Streptomyces sp. neau- } \\
\text { D50 }\end{array}$ & Phytopathogenic fungi & $\begin{array}{l}\text { Zhang et al. } \\
\text { (2014) }\end{array}$ \\
\hline
\end{tabular}


Fig.1 Chemical structures of some compounds from endophytic actinomycetes

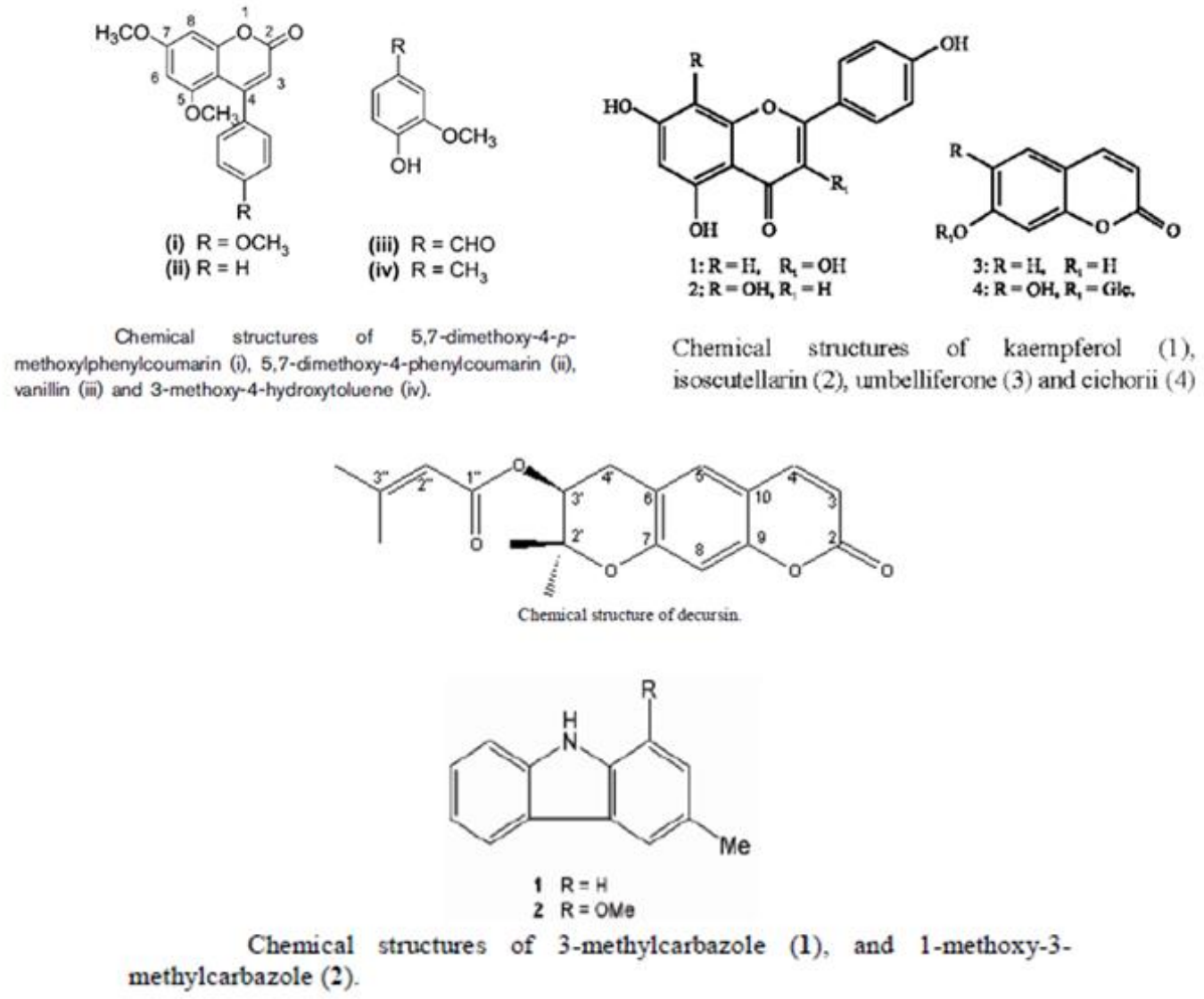

An active fraction, partially purified by TLC and HPLC, from a potent endophytic strain recovered from Euphorbia hirta exhibited inhibitory activity against MRSA strain (Syed et al., 2015). An endophytic actinomycetes strain designated LGMB259 from Vochysia divergens was shown to be potent in inhibiting MRSA (Savi et al., 2015).

\section{Antimicrobial activity of purified compounds from endophytic actinomycetes}

Several compounds have been isolated and characterized from endophytic actinomycetes. These compounds are generally isolated and characterized by chromatographic and spectral techniques (Castillo et al., 2002; Ezra et al., 2004;
Taechowisan et al., 2013). Table 5 shows antimicrobial activity of purified compounds isolated from endophytic actinomycetes. Figure 1 shows the chemical structures of some compounds from endophytic actinomycetes having antimicrobial activity.

In conclusion, it is estimated that $<1 \%$ of all bacteria are currently known and it indicates that millions of microbial species remain to be discovered. The natural condition of plants seems to be in a close interaction with endophytes. The plant endosphere is considered as a complex micro-ecosystem in which different niches can be colonized by different types of microorganisms representing rich sources of novel bioactive metabolites. Endophytes appear to play an important role in ecological systems by shaping plant communities and mediating 
ecological interactions. Endophytes in particular actinomycetes seem promising to increase crop yields, remove contaminants, inhibit pathogens, and produce fixed nitrogen or novel substances. Endophytes are a rich and reliable source of genetic diversity and biological novelty as they produce wide array of compounds having useful activities. More research on the formulation, development of novel technologies and methodologies is needed for employing them in the agricultural, medical and pharmaceutical fields. An extensive research on characterization and identification of the diverse population of endophytic actinobacteria associated with plants also provide greater insight into the plant-endophyte interaction. There is a pressing need for search of new therapeutic drugs, particularly anti-infective compounds due to the rapid increase of resistance in major known pathogens. Therefore, screening and isolation of promising strains of microbes, particularly endophytic actinomycetes, with antimicrobial properties has increased the interest of researchers in both basic and applied fields.

\section{References}

Berdy, J. 2005. Bioactive microbial metabolites. J. Antibiotics, 58: 1-26.

Birber, B., Nuske, J., Ritzau, M., Grafe, U. 1998. Alnumycin, a new naphthoquinone antibiotic produced by an endophytic Streptomyces sp. J. Antibiotics, 51: 381382.

Bunyoo, C., Duangman, K., Nuntagij, A., Thamchaipenet, A. 2009. Characterisation of endophytic actinomycetes isolated from wattle trees (Acacia auriculiformis A. Cunn. ex Benth.) in Thailand. Thai J. Genetics, 2(2): 155-163.

Cao, L., Qiu, Z., You, J., Tan, H., Zhou, S. 2004. Isolation and characterization of endophytic Streptomyces strains from surface-sterilized tomato (Lycopersicon esculentum) roots. Lett. Appl. Microbiol., 39: 425-430.

Castillo, U., Harper, J.K., Strobel, G.A., Sears, J., Alesi, K., Ford, E., Lin, J., Hunter, M., Maranta, M., Ge, H., Yaver, D., Jensen, J.B., Porter, H., Robison, R., Millar, D., Hess, W.M., Condron, M. and Teplow, D. 2003. Kakadumycins, novel anti biotics from, an endophyte of Grevillea pteridifolia. FEMS Microbiol. Lett., 224(2): 183-190.

Castillo, U., Strobel, G., Ford, E., Hess, W., Porter, H., Jensen, J.B., Albert, H., Robison, R., Condron, M.A., Teplow, D.B., Stevens, D. and Yaver, D. 2002. Munumbicins, wide-spectrum antibiotics produced by Streptomyces NRRL 30562, endophytic on Kennedia nigriscans. Microbiol., 148: 2675-2685.

Conti, R., Cunha, I.G.B., Siqueira, V.M., Souza-Motta, C.M., Amorim, E.L.C. and Araujo, J.M. 2012. Endophytic microorganisms from leaves of Spermacoce verticillata (L.): Diversity and antimicrobial activity. J. Appl. Pharmaceutical Sci., 2(12): 17-22.

Dalal, J.M., Kulkarni, N.S. 2014. Antagonistic and plant growth promoting potentials of indigenous endophytic actinomycetes of soybean (Glycine $\max$ (L) Merril). CIBTech J. Microbiol., 3(4): 1-12.

Davies, J., Davies, D. 2010. Origins and evolution of antibiotic resistance. Microbiol. Mol. Biol. Rev., 74(3): 417433.

de Araújo, J.M., da Silva, A.C. and Azevedo, J.L. 2000. Isolation of endophytic actinomycetes from roots and leaves of maize (Zea mays L.). Brazilian Archives of Sci. Technol., 43(4):http://dx.doi.org/10.1590/S151689132000000400016.

Dzidic, S., Suskovic, J., Kos, B. 2008. Antibiotic resistance mechanisms in Bacteria: Biochemical and genetic aspects. Food Technol. Biotechnol., (1): 11-21.

El-Gendy, M.M.A. and EL-Bondkly, A.M.A. 2010. Production and genetic 
improvement of a novel antimycotic agent, saadamycin, against dermatophytes and other clinical fungi from endophytic Streptomyces sp. Hedaya48. J. Industrial Microbiol. Biotechnol., 37(8): 831-841.

El-Shatoury, S., Abdulla, H., El-Karaaly, O., El-Kazzaz, W. and Dewedar, A. 2006. Bioactivities of endophytic actinomycetes from selected medicinal plants in the world heritage site of Saint Katherine, Egypt. Int. J. Bot., 2(3): 307-312.

Ezra, D., Castillo, U.F., Strobel, G.A., Hess, W.M., Porter, H., Jensen, J.B., Condron, M.A.M., Teplow, D.B., Sears, J., Maranta, M., Hunter, M., Weber, B. and Yaver, D. 2004. Coronamycins, peptide antibiotics produced by a verticillate Streptomyces sp. (MSU-2110) endophytic on Monstera sp. Microbiol., 150: 785793.

Gangwar, M., Dogra, S. and Sharma, N. 2011. Antagonistic bioactivity of endophytic actinomycetes isolated from medicinal plants. J. Adv. Laboratory Res. Biol., 2(4): 154-157.

Gangwar, M., Dogra, S., Gupta, U.P. and Kharwar, R.N. 2014. Diversity and biopotential of endophytic actinomycetes from three medicinal plants in India. African J. Microbiol. Res., 8(2): 184-191.

Gayathri, P. and Murulikrishnan, V. 2013. Antimicrobial activity of endophytic actinomycetes isolated from sugarcane and banana plant. Indian Streams Res. J., 3(1).

Ghadin, N., Zin, N.M., Sabaratnam, V., Badya, N., Basri, D.F., Lian, H.H. and Sidik, N.M. 2008. Isolation and characterization of a novel endophytic Streptomyces SUK 06 with antimicrobial activity from Malaysian plant. Asian $J$. Plant Sci., 7: 189-194.

Golinska, P., Wypij, M., Agarkar, G., Rathod, D., Dahm, H. and Rai, M. 2015. Endophytic actinobacteria of medicinal plants: diversity and bioactivity. Antonie van Leeuwenhoek, 108: 267-289.

Gonzalez-Franco, A.C., Robles-Hernandez, L., Nuñez-Barrios, A., Strap, J.L. and
Crawford, D.L. 2009. Molecular and cultural analysis of seasonal actinomycetes in soils from Artemisia tridentate habitat. Int. J. Experimental Bot., 78: 83-90.

Goodfellow, M., Kampfer, P., Busse, H.J., Trujillo, M.E., Suzuki, K.I., Ludwig, W. and Whitman, W.B. 2012. Bergey's manual of systematic bacteriology, $2^{\text {nd }}$ edn, vol 5 The Actinobacteria, Part A, Springer, New York.

Gu, Q., Liu, N., Qiu, D.H., Liu, Z.H. and Huang, Y. 2006. Isolation, classification and antimicrobial activity of endophytic actinomycetes from plant leaves. Wei Sheng Wu Xue Bao, 46(5): 778-82.

Hasegawa, S., Meguro, A., Shimizu, M., Nishimura, T. and Kunoh, H. 2006. Endophytic actinomycetes and their interactions with host plants. Actinomycetologica, 20: 72-81.

Huang, X.L., Zhuang, L., Lin, H.P., Li, J., Goodfellow, M. and Hong, K. 2012. Isolation and bioactivity of endophytic filamentous actinobacteria from tropical medicinal plants. African J. Biotechnol., 11(41): 9855-9864.

Igarashi, Y. 2004. Screening of novel bioactive compounds from plantassociated actinomycetes. Actinomycetologica, 18: 63-66.

Igarashi, Y., Trujillo, M.E., Martinez-Molina, E., Yanase, S., Miyanaga, S., Obata, T. et al. 2007. Antitumor anthraquinones from an endophytic actinomycete Micromonospora lupine sp. nov. Bioorg. Med. Chem. Lett., 17: 3702-3705.

Joseph, B., Sankarganesh, P., Edwin, B.T. and Rai, J.S. 2012. Endophytic Streptomycetes from plants with novel green chemistry. Int. J. Biol. Chem., 6: 42-52.

Kafur, A., Khan, A.B. 2011. Isolation of endophytic actinomycetes from Catharanthes roseus (L.) G. Don leaves and their antimicrobial activity. Iranian J. Biotechnol., 9(4): 302-306.

Kandpal, K.C., Jain, D.A., Kumar, U., Tripathi, R. and Kumar, S.T. 2012. 
Isolation and screening of endophytic actinomycetes producing antibacterial compound from Citrus aurantifolia fruit. European J. Experimental Biol., 2(5): 1733-1737.

Kekuda, P.T.R. 2014. Purification, characterization and pharmacological activities of bioactive metabolites from potent actinomycetes strains. Ph.D thesis, Kuvempu University, India.

Kekuda, P.T.R., Dileep, N., Junaid, S., Rakesh, K.N., Mesta, S.C. and Onkarappa, R. 2013. Biological activities of Streptomyces species SRDP-07 isolated from soil of Thirthahalli, Karnataka, India. Int. J. Drug Development and Res., 5(3): 268-285.

Kekuda, P.T.R., Shobha, K.S. and Onkarappa, R. 2010. Fascinating diversity and potent biological activities of Actinomycete metabolites. J. Pharmacy Res., 3(2): 250256.

Kekuda, P.T.R., Onkarappa, R., Gautham, S.A., Mesta, S.C. and Raghavendra, H.L. 2015. Antimicrobial, antioxidant and cytotoxic activity of Streptomyces species from Western Ghat soils of Karnataka, India. Sci. Technol. Arts Res. J., 4(2): 164-180.

Kumar, S., Krishnan, K. 2012. Cytotoxicity and antioxidant activity of 5-(2,4dimethylbenzyl)pyrrolidin-2-one

extracted from marine Streptomyces VITSVK5 spp. Saudi J. Biol. Sci., 19: 8186.

Li, J., Zhao, G.Z., Chen, H.H., Wang, H.B., Qin, S., Zhu, W.Y., Xu, L.H., Jiang, C.L. and Li, W.J. 2008. Antitumour and antimicrobial activities of endophytic streptomycetes from pharmaceutical plants in rainforest. Lett. Appl. Microbiol., 47(6): 574-580.

Lu, C.H. and Shen, Y.M. 2003. A new macrolide antibiotics with antitumor activity produced by Streptomyces sp. CS, a commensal microbe of Maytenus hookeri. J. Antibiotics, 56: 415-418.

Lu, C.H. and Shen, Y.M. 2004. Two new macrolides produced by Streptomyces sp.
CS. J. Antibiotics, 57: 597-600.

Machavariani, N.G., Ivankova, T.D., Sineva, O.N. and Terekhova, L.P. 2014. Isolation of endophytic actinomycetes from medicinal plants of the Moscow region, Russia. World Appl. Sci. J., 30(11): 15991604.

Mai, N., Matainaho, T., Rai, P.P. and Barrows, L.R. 2013. Antimicrobial activity of endophytes in six medicinal plants collected in the central province, Papua New Guinea. Pacific J. Medi. Sci. 11(2): 57-69.

Minamiyama, H., Shimizu, M., Kunoh, H., Furumai, T., Igarashi, Y., Onaka, H. and Yoshida, R. 2003. Multiplication of isolate R-5 of Streptomyces galbus on rhododendron leaves and its production of cell wall-degrading enzymes. J. General Plant Pathol., 69: 65-70.

Muthiah, B., Stanley, S. and Namasivayam, S.K.R. 2009. Screening of endophytic actinomycetes residing in Eucalyptus globus for antimicrobial activity against human pathogenic bacteria. J. Chemical and Pharmaceutical Sci., 2(2): 154-157.

Nimnoi, P., Pongsilp, N., Lumyong, S. 2010. Genetic diversity and community of endophytic actinomycetes within the roots of Aquilaria crassna Pierre ex Lec assessed by actinomycetes-specific PCR and PCR-DGGE of $16 \mathrm{~S}$ rRNA gene. Biochemical Systematics and Ecol., 38(4): 595-601.

Passari, A.K., Mishra, V.K., Gupta, V.K. Yadav, M.K., Saikia, R. and Singh, B.P. 2015. In vitro and in vivo plant growth promoting activities and DNA fingerprinting of antagonistic endophytic actinomycetes associates with medicinal plants. PLOS ONE, 10(9): e0139468. doi:10.1371/journal.pone.0139468.

Petrini, O., Sieber, T.N., Toti, L. and Viret, O. 1992. Ecology, metabolite production, and substrate utilization in endophytic fungi. Natural Toxins, 1(3): 185-196.

Phuakjaiphaeo, C. and Kunasakdakul, K. 2015. Isolation and screening for inhibitory activity on Alternaria 
brassicicola of endophytic actinomycetes from Centella asiatica (L.) Urban. J. Agri. Technol., 11(4): 903-912.

Piza, A.C.M.T., Hokka, C.O. and de Sousa, C.P. 2015. Endophytic actinomycetes from Miconia albicans (Sw.) Triana (Melastomataceae) and evaluation of its antimicrobial activity. J. Scientific Res. Reports, 4(4): 281-291.

Pullen, C., Schmitz, P., Meurer, K., Bamberg, D.D., Lohmann, S., Franc, S.D.C., Groth, I., Schlegel, B., Möllmann, U., Gollmick, F., Gräfe, U. and Leistner, E. 2002. New and bioactive compounds from Streptomyces strains residing in the wood of Celastraceae. Planta, 216:162-167.

Pujiyanto, S., Lestari, Y., Suwanto, A., Budiarti, S. and Darusman, L.K. 2012. Alpha-glucosidase inhibitor activity and characterization of endophytic actinomycetes isolated from some Indonesian diabetic medicinal plants. Int. J. Pharmacy and Pharmaceutical Sci., 4(1): 327-333.

Qin, S., Li, J., Chen, H., Zhao, G., Zhu, W., Jiang, C., Xu, L. and Li, W. 2009. Isolation, diversity, and antimicrobial activity of rare actinobacteria from medicinal plants of tropical rain forests in Xishuangbanna, China. Appl. Environ. Microbiol., 75(19): 6176-6186.

Qin, S., Xing, K., Jiang, J., Xu, L. and Li, W. 2011. Biodiversity, bioactive natural products and biotechnological potential of plant-associated endophytic actinobacteria. Appl. Microbiol. Biotechnol., 89: 457-473.

Rao, Y.H.C., Rakshith, D. and Satish, S. 2015. Antimicrobial properties of endophytic actinomycetes isolated from Combretum latifolium Blume, a medicinal shrub from Western Ghats of India. Frontiers in Biol., 10(6): 528-536.

Rosenblueth, M. and Martínez-Romero, E. 2006. Bacterial endophytes and their interactions with hosts. Molecular PlantMicrobe Interactions, 19(8): 827-837.

Rossello'-Mora, R. and Amann, R. 2001. The species concept for prokaryotes. FEMS
Microbiol., 25: 39-67.

Roy, S. and Banerjee, D. 2015. Bioactive endophytic actinomycetes of Cinnamomum sp.; Isolation, identification, activity guided purification and process optimization of active metabolite. American J. Microbiol., 6(1): 4.13.

Ryan, R.P., Germaine, K., Franks, A., Ryan, D.J. and Dowling, D.N. 2008. Bacterial endophytes: recent developments and applications. FEMS Microbiol. Lett., 278: $1-9$.

Saadoun, I. and Al-Momani, F. 2000. Activity of North Jordan soil streptomycete isolates against Candida albicans. World J. Microbiol. Biotechnol., 16: 139-142.

Saini, P., Gangwar, M., Kalia, A., Singh, N. and Narang, D. 2016. Isolation of endophytic actinomycetes from Syzygium cumini and their antimicrobial activity against human pathogens. J. Appl. Natural Sci., 8(1): 416-422.

Savi, D.C., Haminiuk, C.W.I., Sora, G.T.S., Adamoski, D.M., Kenski, J., Winnischofer, S.M.B. and Glienke, C. 2015. Antitumor, antioxidant and antibacterial activities of secondary metabolites extracted by endophytic actinomycetes isolated from Vochysia divergens. Int. J. Pharmaceutical, Chem. Biol. Sci., 5(1): 347-356.

Selim, K.A., El-Beih, A.A., AbdEl-Rahman, T.M. and El-Diwany, A.I. 2012. Biology of Endophytic Fungi. Curr. Res. Environ. Appl. Mycol., 2(1): 31-82.

Shenpagam, H.N., Devi, K.D., Sinduja, G. and Sandhya, R. 2012. Isolation of endophytic actinomycetes from medicinal plants and its mutational effect in biocontrol activity. Int. J. Pharmaceutical Sci. Res., 3(11): 4338-4344.

Shirling, E.B. and Gottlieb, D. 1966. Methods for characterization of Streptomyces species. Int. J. Systematic Bacteriol., 16: 313-340.

Singh, M.J. and Padmavathy, S. 2015. Nocardiopsis sp. 5 endophytic to tulsi leaves-Isolation and antimicrobial 
activity. British Microbiol. Res. J., 5(3): 194-202.

Singh, R. and Dubey, A.K. 2015. Endophytic actinomycetes as emerging source for therapeutic compounds. Indo Global J. Pharmaceutical Sci., 5(2): 106-116.

Sreeja, S.J. and Gopal, S.K. 2013. Bioefficacy of endophytic actinomycetes for plant growth promotion and management of bacterial wilt in tomato. Pest Management in Horticultural Ecosystems, 19(1): 63-66.

Strobel, G. and Daisy, B. 2003. Bioprospecting for microbial endophytes and their natural products. Microbiol. Mol. Biol. Rev., 67: 491-502.

Sunaryanto, R. and Mahsunah, A.H. 2013. Isolation, purification, and characterization of antimicrobial substances from endophytic actinomycetes. Makara J. Sci., 17/3. doi: 10.7454/mss.v17i3

Syed, B., Prasad, N.M.N., Rao, Y.H.C., Rakshith, D., Maithri, B., Kavitha, K.S., Azmath, P., Kavitha H.U., Harini, B.P., Kumar, K., Zarei, M. and Satish, S. 2015. Actinomycetic symbionts inhabiting Euphorbia hirta L. with antimicrobial potentials. J. Biol. Active Products from Nature, 5(6): 419-426.

Taechowisan, T. and Lumyong, S. 2003. Activity of endophytic actinomycetes from roots of Zingiber officinale and Alpinia galanga against phytopathogenic fungi. Annals of Microbiol., 53(3): 291298.

Taechowisan, T., Chanaphat, S., Ruensamran, W. and Phutdhawong, W.S. 2013. Antibacterial activity of Decursin from Streptomyces sp. GMT-8; an endophyte in Zingiber officinale Rosc. J. Appl. Pharmaceutical Sci., 3(10): 74-78.

Taechowisan, T., Chuaychot, N., Chanaphat, S., Wanbanjob, A. and Shen, Y. 2009. Antioxidative and inhibitory activity on nitric oxide production of flavonoids from Streptomyces sp. Tc052. J. Pharmacy Res., 2(3): 313-316.

Taechowisan, T., Chuaychot, N., Chanaphat,
S., Wanbanjob, A. and Shen, Y. 2008. Biological activity of chemical constituents isolated from Streptomyces sp. Tc052, an endophyte in Alpinia galanga. Int. J. Pharmacol., 4(2): 95-101.

Taechowisan, T., Lu, C., Shen, Y. and Lumyong, S. 2007. Antitumor activity of 4-arylcoumarins from endophytic Streptomyces aureofaciens CMUAc130. J. Cancer Res. Therapeutics, 3(2): 86-91.

Taechowisan, T., Lu, C., Shen, Y. and Lumyong, S. 2005. Secondary metabolites from endophytic Streptomyces aureofaciens CMUAc130 and their antifungal activity. Microbiol., 151: 1691-1695.

Taechowisan, T., Lu, C., Shey, Y. and Lumyong, S. 2007. In vitro antiinflammatory activity of 4-arylcoumarins from endophytic Streptomyces aureofaciens CMUAc130 in murine macrophage RAW264.7 cells. Natural Product Res., 21(12): 1104-1113.

Taechowisan, T., Wanbanjob, A., Tuntiwachwuttikul, P. and Taylor, W.C. 2006. Identification of Streptomyces sp. Tc022, an endophyte in Alpinia galanga, and the isolation of actinomycin D. Annals of Microbiol., 56(2): 113-117.

Takahashi, Y. and Omura, S. 2003. Isolation of new actinomycetes strains for the screening of new bioactive compounds. $J$. General and Appl. Microbiol., 49: 141154.

Tenover, F.C. 2006. Mechanisms of antimicrobial resistance in bacteria. The American J. Med., 119(6A): S3-S10.

Tanvir, R., Sajid, I. and Hasnain, S. 2014. Larvicidal potential of Asteraceae family endophytic actinomycetes against Culex quinquefasciatus mosquito larvae. Natural Product Res., 28(22): 2048-2052.

Verma, V.C., Gond, S.K., Kumar, A., Mishra, A., Kharwar, R.N. and Gange, A.C. 2009. Endophytic actinomycetes from Azadirachta indica A. Juss.: Isolation, diversity, and antimicrobial activity. Microbial Ecol., 57(4): 749-756.

Vijayan, V.M., Radhakrishnan, M. and 
Balagurunathan, R. 2014. Bioprospecting of endophytic actinomycetes for antiphytofungal activity. Int. J. Chem. Tech. Res., 6(5): 2689-2694.

Wang, X.J., Min, C.L., Yin, Z.C. and Yang, Y. 2014. Isolation and identification of an antimicrobial endophytic actinomycete from Macleaya cordata. Zhong Yao Cai. 37(11): 1947-1950.

Wayne, L.G., Brenner, D.J., Colwell, R.R., Grimont, P.A.D., Kandler, O., Krichevsky, M.I., Moore, L.H., Moore, W.E.C., Murray, R.G.E., Stackebrandt, E., Starr, M.P. and Truper, H.G. 1987. Report of the ad hoc committee on reconciliation of approaches to bacterial systematics. Int. J. Systematic Bacteriol., 37: 463-464.

Yan, L.L., Han, N.N., Zhang, Y.Q., Yu, L.Y., Chen, J., Wei, Y.Z., Li, Q.P., Tao, L., Zheng, G.H., Yang, S.E., Jiang, C.X., Zhang, X.D., Huang, Q., Habdin, X., Hu, Q.B., Li, Z., Liu, S.W., Zhang, Z.Z., He, Q.Y., Si, S.Y. and Sun, C.H. 2010. Antimycin A18 produced by an endophytic Streptomyces albidoflavus isolated from a mangrove plant. $J$. Antibiotics, 63: 259-261.

Zhang, H.W., Song, Y.C. and Tan, R.X. 2006. Biology and chemistry of endophytes $\dagger$. Natural Product Report, 23: 753-771.

Zhang, J., Wang, J.D., Liu, C.X., Yuan, J.H., Wang, X.J. and Xiang, W.S. 2014. A new prenylated indole derivative from endophytic actinobacteria Streptomyces sp. neau-D50. Natural Product Res., 28(7): 431-437.

Zhang, X., Ren, K. and Zhang, L. 2012. Screening and preliminary identification of medicinal plants endophytic actinomycetes used for inhibiting penicillin-resistant Staphylococcus aureus. Int. J. Biol., 4(2): 119-124.

Zhao, G., Li, J., Qin, Y., Miao, C., Wei, D., Zhang, S., Xu, L. and Li, W. 2012. Pseudonocardia antimicrobica sp. nov., a novel endophytic actinomycete associated with Artemisia annua L. (sweet wormwood). J. Antibiotics 65: 469-472.

Zhao, H., Parry, R.L., Ellis, D.I., Griffith, G.W. and Goodacre, R. 2006. The rapid differentiation of Streptomyces isolates using Fourier transform infrared spectroscopy. Vibrational Spectroscropy, 40: 213-218.

Zhao, P.J., Fan, L.M., Li, G.H., Zhu, N. and Shen, Y.M. 2005. Antibacterial and antitumor macrolides from Streptomyces sp. 1s9131. Arch. Pharma. Res., 28: 12281232.

Zhou, H., Yang, Y., Peng, T., Li, W., Zhao, L., Xu, L. and Ding, Z. 2014. Metabolites of Streptomyces sp., an endophytic actinomycetes from Alpinia oxyphylla. Natural Product Res., 28(4): 265-267.

Zin, N.M.,, Sarmin, N.I.M., Ghadin, N., Basri, D.F., Sidik, N.M., Hess, W.M. and Strobel, G.A. 2007. Bioactive endophytic streptomycetes from the Malay Peninsula. FEMS Microbiol. Lett., 274: 83-88.

\section{How to cite this article:}

Prashith Kekuda, T.R., 2016. Isolation, Characterization and Antimicrobial Potential of Endophytic Actinomycetes. Int.J.Curr.Microbiol.App.Sci. 5(7): 100-116. doi: http://dx.doi.org/10.20546/ijcmas.2016.507.008 